\title{
ESTÍMULOS E BARREIRAS PARA O APROVEITAMENTO DE RESÍDUOS DE MADEIRA NA FABRICAÇÃO DE BRIQUETES: ESTUDO DE CASOS
}

Jéssica Mariella Bauer*

Miguel Afonso Sellitto**

RESUMO: A indústria de processamento mecânico da madeira é importante geradora de resíduos lignocelulósicos. Estes são usualmente descartados e se o forem de modo inadequado, podem causar danos ambientais. Dadas as recentes exigências ambientais, empresas do setor têm considerado investimentos no aproveitamento de resíduos como a serragem, cavacos e o pó de madeira para confecção de briquetes. O objetivo deste artigo é identificar os motivos que levam uma empresa de processamento de madeira a aproveitar os resíduos de seu processo produtivo para a produção de briquetes e os fatores que possam dificultar ou facilitar esse processo. O método de pesquisa adotado neste trabalho foi o estudo de casos múltiplos. Analisaram-se duas empresas de beneficiamento de madeira que confeccionam briquetes (B1 e B2) e outras duas empresas que não aproveitam seus resíduos (M1 e M2). Os resultados evidenciam que os principais estímulos para a produção de briquetes são a destinação correta dos resíduos em acordo à legislação e a obtenção de ganhos financeiros superiores à simples venda. As principais barreiras observadas foram a alta sazonalidade do negócio e a baixa informação do mercado consumidor sobre vantagens e benefícios de utilização de briquetes. Os setores não obtêm incentivos fiscais, governamentais ou legais no Brasil. Além disso, assinalou-se a disponibilidade de resíduos próprios e de baixa umidade, já que a aquisição de resíduos de outras empresas, transporte e a exigência de secagem aumentam os custos do processo.

PALAVRAS-CHAVE: Biomassa; Briquetes; Resíduos lignocelulósicos.

\footnotetext{
"Mestre em Engenharia de Produção pela Universidade do Vale do Rio dos Sinos (UNISINOS), São Leopoldo, RS, Brasil.E-mail: jehbauer@hotmail.com

${ }^{*}$ Doutor em Engenharia de Produção, docente permanente do Programa de Pós-graduação em Engenharia de Produção e Sistemas da Universidade do Vale do Rio dos Sinos (UNISINOS), São Leopoldo, RS, Brasil.
} 


\title{
STIMULI AND BARRIERS FOR THE USE OF WOOD RESIDUES IN THE MANUFACTURE OF BRIQUETTES: A CASE STUDY
}

\begin{abstract}
Mechanical wood processing is an important generator of lignocellulose wastes, which are normally discarded. If done incorrectly, serious environmental damage ensues. Due to environmental requirement, sectorial firms are interested in investing in residues, such as sawdust, wood chips and wood powder, for the manufacture of briquettes. Current paper identifies the motive used by a wood processing firm to reuse residues in its production process for the production of briquettes, coupled to factors that impair or facilitate the process. Method comprises a study of multiple cases. Two firms specialized in timber processing and in the manufacture of briquettes (B1 and B2) and two others who failed to do so (M1 and M2) are discussed. Results show that the main stimuli for briquette manufacture are the correct reuse of wastes, complying with legislation, with profits higher than their mere sale. The main impairments are high seasonality of the business and scanty information of the consuming market with regard to the advantages and benefits of briquettes. These sectors do not receive any fiscal benefits from the Brazilian government. Further, availability of residues and low humidity should be underscored since the acquisition of the residues from other companies, transport and drying exigencies increase costs.
\end{abstract}

KEY WORDS: Biomass; Briquettes; Lignocellulose residues.

\section{INTRODUÇÃO}

A madeira e seus resíduos são denominados de biomassa lignocelulósica, composta por macromoléculas de alto e baixo peso molecular, tais como celulose, hemicelulose e lignina, assim como resinas, nutrientes e cinzas, entre outros (CORTEZ; LORA; GÓMEZ, 2008; HERBST, 2011; BORGHI, 2012; OLIVEIRA, 2013). A biomassa origina-se de resíduos animais, vegetais, sólidos urbanos, industriais e florestais e pode ser empregada para geração de energia (CORTEZ; LORA; GÓMEZ, 2008; BUZIN, 2009; SILVA; CARNEIRO; LOPES, 2017) isoladamente ou em conjunto com outros resíduos gerados por processos industriais (MURAKAMI et al., 2015). No Brasil, Felfli et al. (2011) identificaram que as biomassas mais promissoras são os resíduos de madeira, casca de arroz e casca de café. 
Em 2014, o Brasil produziu cerca de 47 milhões de toneladas de resíduos sólidos, sendo que 33,6 milhões de toneladas $(71,5 \%)$ foram gerados pelas atividades florestais e 13,4 milhões de toneladas (28,5\%) pelas atividades industriais. Entre os resíduos florestais destacam-se: serragem, cepilho, maravalha, lenha (aparas, refilos, cascas, roletes) e o pó de serra (DUTRA; NASCIMENTO; NUMAZAWA, 2005; FARAGE, 2009; TUOTO, 2009; DIAS et al., 2012; BARBOSA et al., 2014; GOUVÊA et al., 2017).

A eficiência média das indústrias de processamento mecânico de madeira é de cerca de 55\% (ALMEIDA; SILVA; ANGELO, 2011), o que significa que expressivos montantes de resíduos são gerados nesta indústria. Resíduos de madeira são aproveitados principalmente para queima em caldeira interna ou venda para outras empresas (LIMA; SILVA, 2005; SILVA; CARNEIRO; LOPES, 2017), queima em olarias, utilização como cama de aviários (DALÓLIO et al., 2017) ou no varejo de alimentos (padarias, pizzarias, etc.) (HERBST, 2011).

Os resíduos são uma característica intrínseca da cadeia produtiva da madeira. Constituem-se em passivo ambiental e acarretam importante problema econômico, que é a sua disposição ou destinação final (SCHUTZ; ANAMI; TRAVESSINI, 2010). Nesse sentido, constata-se uma deficiência de soluções sanitárias e ambientalmente próprias para destinação e aproveitamento de resíduos de madeira. As soluções conhecidas se apresentam como incipientes no país, apesar do reconhecido potencial que resíduos possuem como matéria-prima e fonte energética (SELLITTO, 2018).

As empresas da indústria de processamento da madeira muitas vezes não pesquisam fontes alternativas para a utilização de seus resíduos (FARAGE et al., 2013). Mesmo se tratando de resíduo biologicamente degradável, a concentração em local confinado, sua decomposição ou mesmo a queima em incineradores podem causar impactos negativos no ambiente (FAGUNDES, 2003).

A bioenergia proveniente da biomassa é promissora como combustível e possibilita uma solução adequada para a destinação de resíduos (SRIVASTAVA et al., 2014). É uma fonte de energia renovável que pode substituir combustíveis fósseis (GENTIL, 2008; SHARIFI; SWITHENBANK; JAMSA-JOUNELA, 2014; SILVA; CARNEIRO; LOPES, 2017) como, por exemplo, o carvão, o petróleo e o gás natural, não-renováveis (YUSOFF, 2006; EMERHI, 2011). Estas fontes não-renováveis representaram, em 2016, 58,5\% da matriz de energia brasileira, sustentada pelo 
petróleo que contabiliza 44,2\%, enquanto as fontes renováveis representaram 41,5\% no mesmo período (EMPRESA DE PESQUISA ENERGÉTICA, 2017). Importantes indústrias usam biomassa para a plena substituição de combustíveis fósseis (SELLITTO et al., 2013) ou como combustível secundário (LUZ; SELLITTO; GOMES, 2006).

O briquete, também denominado de lenha ecológica (PAULA, 2006; FILIPPETTO, 2008; BRITO; CUNHA, 2009; PONTAROLLI et al., 2013) é um produto obtido pela compactação da biomassa sob alta pressão e em alguns casos temperatura (RAMÍREZ-GÓMEZ et al., 2014). No processo, provoca-se um incremento térmico entre $100{ }^{\circ} \mathrm{C}$ a $200{ }^{\circ} \mathrm{C}$ no resíduo e na lignina, elemento natural da madeira, que se plastifica atuando como elemento aglomerante das partículas. Para que ocorra a densificação, é necessário que a umidade dos resíduos seja de 8\% a 15\% (CARVALHO; BRINCK, 2004; FARAGE, 2009; SILVA, 2007; TAVARES, 2013).

A biomassa utilizada é oriunda principalmente de serrarias, marcenarias e da indústria de móveis e esquadrias (QUIRINO, 2005; GENTIL, 2008; GONÇALVES, 2010; DIAS et al., 2012). O briquete obtido é um biocombustível sólido, em forma de blocos cilíndricos compactados e densificados (BORGHI, 2012; DANTAS; SANTOS; SOUZA, 2012; ROY; CORSCADDEN, 2012).

Diversas são as vantagens atribuídas ao briquete, e que foram amplamente estudadas na academia, sejam elas operacionais, logísticas, energéticas ou ambientais (DIAS et al., 2012). O briquete gera pouca fumaça, cinza e fuligem em relação à madeira bruta e aos resíduos de madeira como a serragem (EMERHI, 2011; PANWAR; BRASAD; WASEWAR, 2011; DANTAS; SANTOS; SOUZA, 2012; PARASHAR; SINGH, 2014). O seu uso oferece benefícios socioeconômicos, tais como geração de emprego e renda para populações vulneráveis e sujeitas a fluxos migratórios e é uma alternativa para combustíveis fósseis, cujos preços podem oscilar de modo inesperado e intenso (DIAS et al., 2012; MWAMPAMBA; OWEN; PIGAHT, 2013). A briquetagem não só elimina resíduos problemáticos e onerosos, como também favorece o processo de geração de calor em caldeiras e fornos, pois permite que os mesmos operem em temperatura mais uniforme do que outros combustíveis e alcance maior temperatura de queima (CAETANO; DEPIZZOL; REIS, 2017).

Muitas pesquisas estudaram as propriedades e características dos briquetes, 
tanto mecânicas como energéticas, tais como: resistência à compressão, ao impacto, à abrasão, à penetração de água, estudos dos teores de materiais voláteis, cinzas e carbono fixo, poder calorífico, densidade, porosidade, entre outros (QUIRINO; BRITO, 1991; BUZIN, 2009; PANWAR; BRASAD; WASEWAR, 2011; SCHUTZ; ANAMI; TRAVESSINI, 2010; PROTÁSIO et al., 2012; ZERBINATTI et al., 2014; GOUVÊA et al., 2017). Todavia, observa-se que as empresas de processamento mecânico da madeira têm dado menos importância ao aproveitamento de resíduos (DIAS, 2012; GOUVÊA et al., 2017).

O objetivo deste artigo é identificar os motivos que levam uma empresa de processamento de madeira a aproveitar os resíduos de seu processo produtivo para a produção de briquetes e os fatores que possam dificultar ou facilitar esse processo.

\section{MATERIAL E MÉTODOS}

O método de pesquisa foi o estudo de caso múltiplo. A escolha do método justifica-se por tratar-se de um tema atual, em que se busca a compreensão em profundidade das razóes para que algumas empresas da indústria de processamento mecânico de madeira, geradoras de grande quantidade de resíduos, optem ou não por aproveitá-los como briquetes. $\mathrm{O}$ estudo é relevante, pois avança na área de aproveitamento de resíduos de madeira, com implicações tanto econômicas como ambientais. $\mathrm{O}$ estudo é de filiação múltipla, pela necessidade da realização de comparações entre duas realidades distintas, de empresas que aproveitam seus resíduos no formato de briquetes e outras que não o fazem, em contextos diferentes. Quanto à categorização, a pesquisa é explicativa: buscam-se razões que expliquem o objeto de pesquisa. A questão de pesquisa é: porque algumas empresas da indústria de processamento da madeira usam seus resíduos para a confecção de briquetes e outras não? Quando a questão de pesquisa é do tipo porque, o método do estudo de caso é adequado (YIN, 2010).

Foram analisadas duas empresas de beneficiamento da madeira que confeccionam briquetes a partir de resíduos (B1 e B2) e duas que não o fazem (M1 e M2). Todas se localizam nos Vales do Paranhana e Sinos, no Rio Grande do Sul. 
Foram entrevistados os gestores de M1, M2 e B1, e o representante comercial de B2. Realizou-se análise de documentos internos e observação dos processos produtivos das empresas. O Quadro 1 apresenta o protocolo com as questões que nortearam as entrevistas. $\mathrm{O}$ protocolo foi construído com base na revisão de literatura que foi revisada para este estudo.

Quadro 1. Protocolo de pesquisa

\begin{tabular}{|c|}
\hline Caracterização da Empresa e Gestão de Resíduos de Madeira \\
\hline $\begin{array}{ll}\text { - } & \text { Processo produtivo } \\
\text { - } & \text { Matéria-prima (espécie de madeira) } \\
\text { - } & \text { Capacidade produtiva } \\
\text { - } & \text { Tipos e quantidades de resíduos gerados } \\
\text { - } & \text { Opções disponíveis para destinação dos resíduos } \\
\text { - } & \text { Preço de venda } \\
\text { - } & \text { Dificuldades de gestão }\end{array}$ \\
\hline Motivações e Barreiras para Briquetagem \\
\hline $\begin{array}{l}\text { - A empresa já pesquisou alternativas de destinação dos resíduos? Há interesse organizacional } \\
\text { - } \quad \text { Com aproveitar seus resíduos? } \\
\text { - } \quad \text { Quais os motivadores para investir na briquetagem de seus resíduos (econômico, ecológico, } \\
\text { legal, de imagem corporativa)? } \\
\text { - O que limita a produção de briquetes? Quais as barreiras e inibidores para investir neste } \\
\text { segmento (econômico, ecológico, legal, de imagem corporativa)? }\end{array}$ \\
\hline
\end{tabular}

Algumas empresas da indústria medem a produção em $\mathrm{m}^{3}$, outras medem em toneladas, portanto é comum encontrarem-se as duas unidades. A indústria usa as seguintes relações aproximadas: $0,96 \mathrm{~m}^{3}$ por tonelada para madeira e $4 \mathrm{~m}^{3}$ por tonelada para resíduo, devido à umidade. O Quadro 2 apresenta a descrição das empresas e resíduos de madeira gerados. A tabela descreve a empresa, o tipo de resíduo gerado, a faixa de umidade observada nos resíduos gerados, o volume mensal de resíduos gerados (M1 e M2 medem em m³ , B1 e B2 medem em toneladas, porém, por coerência e facilidade de comunicação, neste artigo padronizou-se o uso de $\mathrm{m}^{3}$ ), a destinação e preços típicos obtidos nas operações estudadas. 
Quadro 2. Descrição das empresas e resíduos de madeira gerados

\begin{tabular}{|c|c|c|c|c|}
\hline & M1 & M2 & B1 & B2 \\
\hline Empresa & $\begin{array}{l}\text { Empresa familiar de } \\
\text { esquadrias fundada } \\
\text { em } 1960, \text { com } 90 \\
\text { funcionários. Usa } \\
\text { madeira de Eucalyptus } \\
\text { (60-70\%), bem como } \\
\text { angelim (Dinizia excel- } \\
\text { sa Ducke), garapeira } \\
\text { (Apuleiamolaris) e } \\
\text { louro freijó (Cordia } \\
\text { goeldiana Huber). }\end{array}$ & $\begin{array}{l}\text { Fundada em } 1994, \\
\text { conta com } 25 \text { fun- } \\
\text { cionários. Beneficia } \\
\text { Pinus elliottii }(90 \%) \\
\text { e Eucalyptus }(10 \%) \text {. } \\
\text { Seus principais } \\
\text { produtos são: meia } \\
\text { cana, rodapés, } \\
\text { forros, assoalhos, } \\
\text { paredes, caibros, } \\
\text { ripas, entre outros. } \\
\text { Beneficia } 10.800 \text { m³ }^{3} \\
\text { de madeira (11 mil } \\
\text { toneladas) por ano. }\end{array}$ & $\begin{array}{l}\text { Empresa familiar } \\
\text { de esquadrias } \\
\text { fundada em } 1951 . \\
\text { Possui } 83 \text { funcio- } \\
\text { nários. }\end{array}$ & $\begin{array}{l}\text { Desde } 1990 \text { é refe- } \\
\text { rência na América } \\
\text { Latina na produ- } \\
\text { ção de móveis de } \\
\text { madeira maciça } \\
\text { de alta qualidade } \\
\text { e design de luxo. } \\
\text { Opera } 80 \text { lojas } \\
\text { exclusivas com as } \\
\text { espécies: Eucalyp- } \\
\text { tus, jequitibá (Ca- } \\
\text { rinianalegali), teca } \\
\text { (Tectonagrandis) e } \\
\text { caixeta (Tabebuia } \\
\text { cassinoides) }\end{array}$ \\
\hline $\begin{array}{l}\text { Tipos de Resí- } \\
\text { duos }\end{array}$ & Serragem e cavaco. & $\begin{array}{l}\text { Serragem, cavaco e } \\
\text { maravalha. }\end{array}$ & $\begin{array}{l}\text { Lenha, cavaco, } \\
\text { serragem e pó de } \\
\text { madeira. }\end{array}$ & $\begin{array}{l}\text { Serragem, cavaco, } \\
\text { pó de serra e pó } \\
\text { de lixa. }\end{array}$ \\
\hline Umidade & $\begin{array}{l}\text { Serragem e cavaco } \\
\text { secos (10-15\% de } \\
\text { umidade) e verdes (20- } \\
30 \% \text { de umidade) }\end{array}$ & $\begin{array}{l}13 \text { a } 17 \% \text { de umi- } \\
\text { dade }\end{array}$ & $\begin{array}{l}\text { Máximo aceitável é } \\
\text { de } 12 \% \text {. A empresa } \\
\text { possui estufa para } \\
\text { secagem da madei- } \\
\text { ra bruta }\end{array}$ & $\begin{array}{l}12 \text { a } 15 \% \text { de umi- } \\
\text { dade }\end{array}$ \\
\hline $\begin{array}{l}\text { Geração média } \\
\text { de Resíduos }\end{array}$ & $\begin{array}{l}1.600 \mathrm{~m}^{3} \text { ( } 400 \text { tone- } \\
\text { ladas) de resíduos } \\
\text { mensais, sendo } 600 \mathrm{~m}^{3} \\
\text { de serragem e de } 800 \mathrm{a} \\
1.000 \mathrm{~m}^{3} \text { de cavaco }\end{array}$ & $\begin{array}{l}\text { Até } 640 \mathrm{~m}^{3}(120 \mathrm{a} \\
160 \text { toneladas }) \text { de } \\
\text { resíduos mensais }\end{array}$ & $\begin{array}{l}720 \mathrm{~m}^{3} \text { (180 tone- } \\
\text { ladas) de resíduos } \\
\text { mensais }\end{array}$ & $\begin{array}{l}800 \mathrm{~m}^{3} \text { (200 tone- } \\
\text { ladas) de resíduos } \\
\text { mensais }\end{array}$ \\
\hline Destinação & $\begin{array}{l}\text { Cerâmicas do Vale } \\
\text { do Caí }\end{array}$ & $\begin{array}{l}\text { Empresas da região } \\
\text { da Serra Gaúcha e } \\
\text { Vale do Taquari }\end{array}$ & $\begin{array}{l}\text { Iniciou o processo } \\
\text { de briquetagem em } \\
2013\end{array}$ & $\begin{array}{l}\text { Iniciou a brique- } \\
\text { tagem em } 2010 . \\
\text { Antes, aviários e } \\
\text { laticínios recebiam } \\
\text { resíduos de mara- } \\
\text { valha }\end{array}$ \\
\hline Preço de venda & $\begin{array}{l}\mathrm{R} \$ 14 / \mathrm{m}^{3} \text { (ano base } \\
2015) ; \\
\mathrm{R} \$ 7-8 / \mathrm{m}^{3} \text { (ano base } \\
2010 / 2011)\end{array}$ & $\begin{array}{l}\mathrm{R} \$ 50 / \text { tonelada } \\
\text { (maravalha) e } \mathrm{R} \$ 25 / \\
\text { tonelada (cavacos). }\end{array}$ & $\begin{array}{l}\text { Antes da brique- } \\
\text { tagem era } \mathrm{R} \$ 25 / \\
\text { tonelada. Após, é } \\
\text { de } \mathrm{R} \$ 300 / \text { tonelada } \\
\text { e } \mathrm{R} \$ 15 \text { o saco de } \\
\text { rafia de } 30 \mathrm{~kg}\end{array}$ & $\begin{array}{l}\text { R\$ 300-330/tone- } \\
\text { lada }\end{array}$ \\
\hline
\end{tabular}




\section{RESULTADO E DISCUSSÃO}

O Quadro 3 foi construído segundo as entrevistas e apresenta a cadeia percorrida pelos resíduos e as destinações observadas no estudo.

Quadro 3. Fluxograma dos resíduos de madeira

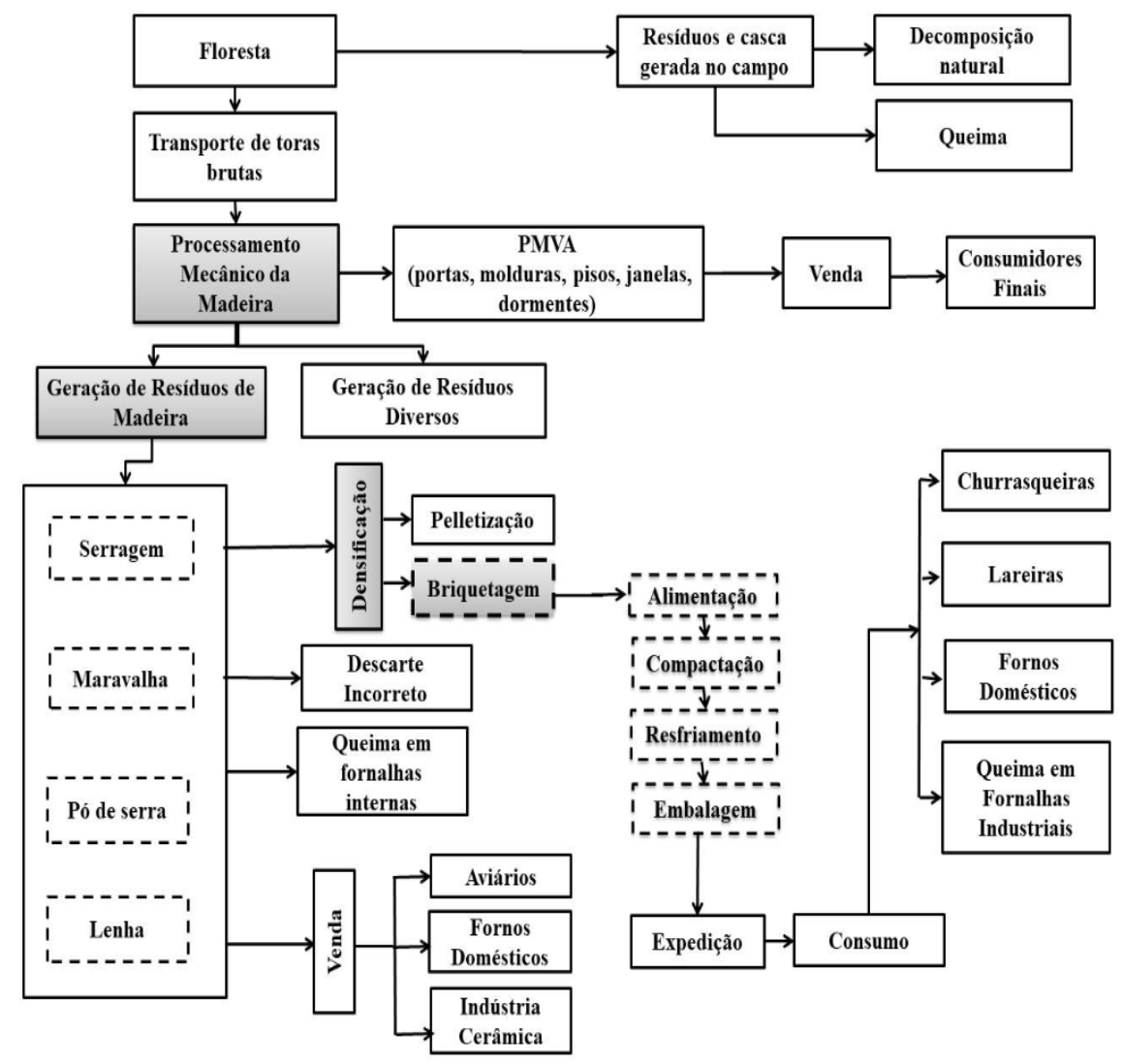

Inicia-se pelo primeiro ponto do protocolo o processo produtivo. Após a madeira bruta ser extraída da floresta ela passa por processamento mecânico, processo no qual são produzidas chapas de madeira reconstituída, partículas, lâminas, compensado e PMVA (Produtos de Maior Valor Agregado) como, por exemplo, portas, molduras, pisos, janelas, dormentes, entre outros. Esta atividade é responsável pela geração de consideráveis montantes de resíduos de madeira entre 
a serragem, maravalha, o pó de serra e a lenha os quais usualmente não apresentam uma finalidade específica de aproveitamento. Entre as opções de destinação apresentam-se opções de aproveitamento como a venda e utilização em aviários, fornos e cerâmicas, a queima direta em fornalhas ou a densificação, em que o resíduo é aproveitado no formato de briquetes ou pellets e é reintroduzido ao ciclo de negócios. Ou é descartado incorretamente no solo o que pode causar impactos ambientais negativos.

O segundo ponto são os produtos e serviços. As empresas analisadas são do ramo de esquadrias (M1 e B1), beneficiamento de madeira (M2) e moveleira (B2). Os principais produtos ofertados por M1 e M2 são esquadrias como portas (de abrir, pivotantes, de garagem, internas e sacadas), janelas, maxiares e vitros. A empresa B2, moveleira, atua com produtos de alto padrão, tais como: mesas, cadeiras, camas, cabeceiras, aparadores, arcas, balcões, banquetas, bases de mesas, camas, escrivaninhas, chaises, cômodas, cristaleiras, estantes, espelhos, quadros, penteadeiras, racks, sofás, poltronas e puffs.

O terceiro ponto é o tipo de matéria-prima (espécie de madeira). As espécies de resíduos predominantes gerados pelas empresas analisadas foram: Eucalyptus, angelim (Dinizia excelsa Ducke), garapeira (Apuleiamolaris), jequitibá (Carinianalegali), teca (Tectonagrandis) e caixeta (Tabebuia cassinoides); B1, B2 e M1; enquanto M2 trabalha com madeira de Eucalyptus spp. e Pinus elliottii.

O quarto ponto é capacidade produtiva. M1 e M2 possuem capacidade de processamento de $2.200 \mathrm{~m}^{3}$ e $10.800 \mathrm{~m}^{3}$ de madeira por mês, respectivamente. B1 e B2 possuem capacidade de processamento mensal de $300 \mathrm{~m}^{3}$ e $1.000 \mathrm{~m}^{3}$ de madeira para a fabricação de esquadrias e peças de mobiliário respectivamente.

O quinto ponto são os tipos e quantidades de resíduos gerados. M1 mensalmente gera em média $600 \mathrm{~m}^{3}$ de serragem e entre 800 e $1.000 \mathrm{~m}^{3}$ de cavaco nas máquinas perfilhadoras, tupias e furadeiras. M2 mensalmente gera em média 640 $\mathrm{m}^{3}$ de resíduos de serragem (aproximadamente 80\%), cavaco (aproximadamente 10\%) e maravalha (aproximadamente 10\%). B1 mensalmente gera em média $720 \mathrm{~m}^{3}$ de resíduos de lenha, cavaco, serragem e pó de madeira. B2 mensalmente gera em média $700 \mathrm{~m}^{3}$ de resíduos de serragem, cavaco, pó de serra e pó de lixa.

O sexto ponto são as opções disponíveis para destinação dos resíduos. M1 
destina a olarias e cerâmica para a produção de tijolos e tavelas. Os resíduos são usados em fornos para secagem do produto final com destinação para o Vale do Caí no Rio Grande do Sul. M2 também destina a olarias e cerâmicas da região da Serra Gaúcha e Vale do Taquari no Rio Grande do Sul. B1 produz briquetes desde 2013, quando adquiriu a máquina briquetadora de uma terceirizada para a qual até então destinava seus resíduos. Por fim, B2 também produz briquetes de madeira desde 2010. Anteriormente os resíduos de maravalha eram encaminhados para aviários e o pó de serra para queima in natura em indústrias de laticínios.

O sétimo ponto é o preço de venda. Em M1 a comercialização é de R\$14/ $\mathrm{m}^{3}$ (ano base 2015), contudo M1 já revendeu o resíduo a uma média de $\mathrm{R} \$ \mathbf{7 - 8}$ / $\mathrm{m}^{3}$ (ano base 2010/2011). Os custos de gestão da serragem e cavacos é em média $50 \%$ do valor de venda, dispendidos em armazenagem, picagem, empilhadeira, dois funcionários, exaustor que funciona 24 horas com motor de 100 CV e a manutenção do mesmo. M2 comercializa a maravalha a $\mathrm{R} \$ 50,00 /$ tonelada para aviários. Já o cavaco passa por picador específico para redução de tamanho da partícula. Este resíduo é comercializado a $\mathrm{R} \$ 25,00 /$ tonelada. Já a empresa B1 comercializa o briquete inteiro a $R \$ 300,00 /$ tonelada e $R \$ 15,00$ o saco de rafia de $30 \mathrm{~kg}$, ao passo que o briquete quebrado é comercializado a $\mathrm{R} \$ 200,00 /$ tonelada e $\mathrm{R} \$ 8,00$ o saco de rafia, preços estabelecidos pelo mercado consumidor. Em comparação os resíduos a granel que eram comercializados por B1 a R\$25,00/tonelada. Quanto à empresa B2 esta vende seus briquetes a valores entre $\mathrm{R} \$ 300,00$ a $\mathrm{R} \$ 330,00$ a tonelada.

$\mathrm{O}$ oitavo e último ponto são as dificuldades de gestão. M1 apontou a dificuldade de venda dos resíduos a granel no mercado e o oligopólio de empresas que adquirem os mesmos. Apontou ainda casos de paralisação da produção por falta de veículos de transporte para coleta dos resíduos e poucos compradores. Além disso, M1 salientou a necessidade de parceria com outras organizações, assim como a obtenção de um consumidor/destinatário interessado na aquisição de todo montante de resíduo produzido. Ou seja, há dificuldade de parceria com um destinatário que compre as diversas espécies de madeira e de distintos índices de umidade. O oligopólio de compradores também foi apontado por M2. Para B1, as principais dificuldades são a lotação de silos e paradas na produção pela demora na coleta dos resíduos, riscos de acidentes por rompimento do silo e vazamento e risco 
de incêndio. B2 apontou somente o baixo ganho econômico na venda dos resíduos, portanto, optou pela produção de briquetes.

Passa-se à análise das respostas. Quanto ao interesse das empresas em pesquisar alternativas de destinação dos resíduos ou aproveitar os mesmos, todas as empresas estudaram alternativas como a produção de pellets e briquetes. M1 e M2 apontaram o interesse em destinar corretamente os resíduos e aumentar os retornos de sua comercialização. M1 já investiu 300 mil reais em picadores e silos. A empresa M1 buscou alternativas em feiras (Curitiba - Paraná) e também visitou picadores e empresas de peletização e briquetagem como formas de aumentar o valor de venda do resíduo. A oferta elevada dificulta a venda, que ocorre a valores irrisórios. M1 estudou alternativas entre 2003 e 2005. Em visita à feira de Hannover (Alemanha), a empresa conheceu a produção de pellets e percebeu vantagens no caso dos pellets de madeira, dada a automação do processo e o poder calorífico de 10 a 11 vezes superior à da serragem in natura. No caso dos pellets, o mercado é a exportação (Europa), que já possui caldeiras específicas para queima do produto. Mas, até o momento, M1 optou por não investir no produto.

M2 iniciou estudos de fontes extras de destinação de resíduos entre os anos de 2011 e 2012. M2 pesquisou alternativas como a densificação de seus resíduos, no formato de pellets e briquetes, mas, optou por buscar parcerias no ano de 2013 e desenvolveu contratos de longo prazo com duas empresas as quais destinam seus resíduos a granel a aviários e olarias. A oportunidade de investimento em briquetes originou-se da necessidade de destinação de resíduos. A oportunidade ocorreu no momento em que se esgotaram as fontes consumidoras do mesmo, por exemplo, cerâmicas da região, e uma empresa produtora de briquetes comprava os mesmos.

B1 iniciou o processo de briquetagem no ano de 2013 quando adquiriu a máquina briquetadora da respectiva empresa para a qual destinava seus resíduos. Previamente, B1 elaborou um plano de negócios e realizou benchmarking.

B2 iniciou o investimento em briquetes no ano de 2010. A empresa foi incentivada por possuir matéria-prima abundante e por visar a obtenção de maior ganho financeiro, assim como destinar corretamente os seus resíduos. A empresa investiu em um mercado que migrava para alternativas mais sustentáveis, como a biomassa. Antes da instalação da briquetagem, B2 realizou benchmarking em organizações localizadas na Europa e em São Paulo. Obteve maior conhecimento 
do produto em uma feira internacional realizada anualmente em Hannover na Alemanha.

Quanto aos motivadores para investir na briquetagem de resíduos, M1 apontou estímulos como o aumento no valor de venda do resíduo e destinação adequada. Como motivação para possibilidade do investimento em briquetes, M1 aponta ter estudado a possibilidade de vender por maior valor os resíduos, simplificar a gestão, e reduzir o risco de o ciclo de produção de esquadrias paralisar e faltar veículos para retirada dos resíduos. Indicou também o incentivo por parte dos consumidores à redução de demanda por lenha e uso de produtos ecologicamente corretos.

Para M2, a motivação em investir em briquetes decorre da estagnação da demanda de maravalha no mercado e por receio de paralisação de sua atividade principal, devido a: dificuldades no escoamento de resíduos; falta de opções de venda; necessidade de destinação adequada às regulamentações; preço de venda considerável; averiguação de outro cliente comprador da maravalha em específico; consumo em padarias, pizzarias e demandantes de biomassa; e interesse em ganho econômico sobre os resíduos.

Para B1, o mercado se tornou atrativo após a redução da queima de lenha por estabelecimentos como pizzarias localizadas no Estado de São Paulo em decorrência da proliferação de roedores e insetos que atacam a lenha e a maior rigor no controle fitossanitário. Entre os estímulos, B1 ressalta, além do exposto, a adequação à PNRS (Política Nacional dos Resíduos Sólidos) com descarte adequado de resíduos, o fim da dependência de terceiros para a retirada do material, destinação sustentável, não gerar pó de serragem e não contaminar o ar por descarga de caminhões. B1 melhorou sua imagem no mercado e participou de campanhas de destinação de resíduos e eventos municipais, como a Semana do Meio Ambiente.

B2 passou a investir no segmento de briquetes por perceber no mercado a busca de utilização de alternativas da biomassa e a PNRS implantada em 2010. A Política Nacional de Resíduos Sólidos estabeleceu como instrumento de desenvolvimento econômico e social a implantação de sistemas de logística reversa, imputando a responsabilidade pós-consumo aos fabricantes, importadores, distribuidores, comerciantes e consumidores. A Lei 12.305/10 disciplina a coleta, o destino final e o tratamento de resíduos, além de estabelecer diretrizes para reduzir 
a geração de lixo e combater o desperdício de materiais descartados (BRASIL, 2010).

Ademais, aos usuários de lenha é exigida licença ambiental pelo IBAMA (Instituto Brasileiro do Meio Ambiente e dos Recursos Naturais Renováveis) o que vem por elevar nos últimos anos o preço final de comercialização do produto.

Para B2, ocorrem avanços quanto ao destino adequado de resíduos e prevenção à contaminação ambiental. B2 também aponta vantagem no sentido de ganho financeiro para o consumidor que adquire o produto, e o emprega como substituto da lenha. B2 afirma que é um produto com maior poder calorífico, que permite o ganho de créditos de carbono. O briquete torna desnecessário um sistema braçal de armazenagem como no caso da lenha, ou a manutenção de um grande espaço de pátio destinado exclusivamente para armazenagem. Reduz-se a sujeira no pátio por perda e desprendimento de material, já que o briquete é armazenado em big bags, facilita-se o recolhimento e translado do pátio-caldeira para queima.

Portanto, o produto exige menor demanda de mão de obra para transporte interno. No caso da lenha, dispende-se tempo entre carregamento e descarregamento de caminhões e entre o pátio e a caldeira. Enquanto com os briquetes, pode-se utilizar de paleteiras e empilhadeiras. Ademais, o produto é entregue Just-in-Time (JIT) conforme as necessidades do cliente em sua fornalha, o que permite a redução dos volumes mantidos em estoque e ocupação de pátio. Por fim, B2 confirma ganhos de imagem no mercado, adequação com a legislação e responsabilidade ambiental.

Diversos fatores limitam a produção e investimento em briquetes. Em primeiro lugar quanto às características da matéria-prima (resíduo), a geração nas empresas foi de cavacos, maravalha, serragem e pó de madeira. $\mathrm{O}$ acondicionamento destes é feito diretamente em silos por meio de sistema interno de exaustão, com exceção de M2, que acondiciona cavacos verdes (umidade de 20 a 30\%) em caixas de quatro metros cúbicos, que primeiramente passam por picador para redução de granulometria, e em seguida seguem para o silo da empresa.

Em relação à caracterização dos resíduos, em M1 os mesmos são secos, e apresentam entre 10 a 15\% de umidade semelhante a M2 em que a umidade varia entre 13 a 17\%. Como B1 e B2 contam com estufas, o máximo observado é de 12\% (B1) e de 12 a 15\% de umidade (B2). Para a briquetagem de resíduos de madeira, Filippetto (2008) ressalta que a umidade elevada é característica indesejada, pois pode provocar explosões quando da prensagem pela formação de vapor. 
B1 e B2 confirmaram a necessidade de baixa umidade. A aquisição de material de terceiros por B1 segue esta limitação: a umidade pode elevar-se, mas em baixo índice. Os resíduos utilizados para briquetagem em B1 e B2 confirmam as recomendações de Gentil (2008), o autor afirma que a umidade dos resíduos para briquetagem deve estar entre 5 e 15\%. B1 e B2 apontaram que umidade mais elevada é indesejada e inviabiliza o processo de briquetagem.

B1 aponta que é viável a briquetagem quando da não necessidade de secar a matéria-prima, ou seja, a madeira bruta foi seca em estufas, e não no tempo. $\mathrm{O}$ ideal é que a empresa possua material próprio (resíduos) em volume suficiente para iniciar a operação de briquetagem. A aquisição de resíduos de outras empresas e os custos de transporte deste material de elevado volume e baixo peso a longas distâncias encarecerá o produto e sua competitividade no mercado. Tal aspecto foi mencionado por Barbosa et al. (2014), Dias et al. (2012), Gentil (2008), Felfli et al. (2011) e Tavares (2013), que apontaram como gargalo a logística para aquisição de uma matéria-prima, volumosa e pouco densa, e que deve estar disponível em localização próxima para não onerar custos de transporte.

B1, B2 e M2 mencionaram também a necessidade de possuir resíduos de espécies adequadas e na granulometria correta. Dias et al. (2012) apontam que as dimensões aceitáveis dos resíduos são entre 6 e $10 \mathrm{~mm}$ sendo que o tamanho das partículas apresenta influência na densidade do produto final. Pode ser necessária a trituração em alguns casos, o que torna mais custoso o produto final. Nesse sentido, conforme a matéria-prima, as empresas elencaram ser necessário (o que não é o caso de B1 e B2) a aquisição de trituradores e secadores para diminuir o tamanho das partículas e/ou reduzir o teor de umidade dos resíduos (FILIPPETTO, 2008). A espécie de pinus, tanto na visão de B1 como de B2, forma um briquete de baixa qualidade e que pode danificar as fornalhas devido à resina que a constitui; nesse sentido, Rosário (2011) aponta que a densidade mais baixa do material produzirá um briquete de menor poder calorífico.

Para M1, B1 e B2, outro entrave é a falta de informação dos consumidores, o que concorda com os estudos de Dantas, Santos e Souza (2012), Emerhi (2011) e Rosário (2011). Portanto, é essencial, conforme B1 e B2, conscientizar os mesmos como no caso da utilização do briquete em diferentes fornalhas. Morais (2007) afirma que os briquetes têm uma propensão para quebrar durante a combustão. 
Dependendo do tamanho podem passar através da grelha ou mesmo bloquear a grelha, o que reduz seu desempenho, e os clientes devem ser orientados.

M1, M2 e B2 apontaram ser elevado o investimento na máquina briquetadora (BHATTACHARYA; SETT; SHRESTHA, 2007). M1 e B1 também ressaltam o elevado custo de energia elétrica para o funcionamento do maquinário. Assim, atualmente, os investidores optam por máquinas usadas, de menor preço, acelerando-se o retorno do investimento, como foi o caso de B1 (ROSÁRIO, 2011).

Para M1, M2, B1 e B2 neste segmento os impostos são elevados como ICMS (17\%), PIS e COFINS $(9,25 \%)$ e tal fator também foi observado por Bhattacharya, Sett e Shrestha (2007). Por fim, M2, B1, B2 apontaram como barreiras a falta de incentivos governamentais, o que também foi apontado por Mwampamba et al. (2013).

Como incentivos, foi unânime o aumento de ganho financeiro na venda e na destinação adequada dos resíduos. B1 e B2 isoladamente apontaram o cumprimento de regulamentações e incentivos como a PNRS; e a exigência de obtenção de licenças ambientais para os usuários de lenha o que é dispensado pelos briquetes conforme observação de Caetano, Depizzol e Reis (2017).

B1 afirmou que o controle fitossanitário sobre roedores atraídos por resíduos a granel também incentiva a adoção de briquetes. B2 afirma o espaço livre nos pátios e a limpeza propiciada pelo produto, o que também foi verificado por autores como Gentil (2008) e Rosário (2011).

B2 fez uma simulação de retorno financeiro, apoiada pelos pesquisadores. Segundo a empresa, o investimento realizado pode ser atualizado em $\mathrm{R} \$ 800.000,00$ e a lucratividade média anual após os impostos pode ser estimada em $R \$ 144.000,00$. Para cinco anos de operação, a TIR (taxa interna de retorno) é de 10\%; para 10 anos, TIR $=16 \%$; para 15 anos, TIR $=20 \%$. Todos os cenários são positivos e ajudam a justificar financeiramente o investimento, que já dá resultado há oito anos.

Os resultados das entrevistas apontam para fatores diversos que podem e devem ser organizados segundo eixos principais. Foram usados os eixos: econômico, ecológico, legal, imagem de mercado, logística, e social. A Tabela 2 sintetiza estímulos e barreiras para o investimento em briquetes verificados, organizadas segundo os eixos supracitados. 
Tabela 2. Síntese dos estímulos e barreiras para a produção de briquetes

\begin{tabular}{|c|c|c|}
\hline Fatores & Estímulos & Barreiras \\
\hline Econômico & $\begin{array}{l}\text { Elevação de receitas. Preço de venda supe- } \\
\text { rior aos resíduos a granel. Independência } \\
\text { de compradores terceirizados e oligopólio } \\
\text { de empresas. Elevado poder calorífico dos } \\
\text { briquetes. Diversidade de aplicações dos } \\
\text { produtos (caldeiras, pizzarias, padarias, } \\
\text { olarias, cerâmicas, indústrias, etc.) }\end{array}$ & $\begin{array}{l}\text { Elevado custo de aquisição do equipa- } \\
\text { mento. Obtenção de picador (tamanho } \\
\text { do resíduo). Sazonalidade forte no } \\
\text { período do verão e necessidade de } \\
\text { depósito para estocagem do produto. } \\
\text { Consumo de energia é elevado no caso } \\
\text { de a matéria-prima for de alta umidade } \\
\text { e precisar passar por secagem. Elevada } \\
\text { concorrência de derivados do petróleo } \\
\text { e lenha. Poder calorífico depende da } \\
\text { umidade do resíduo. Custos elevados } \\
\text { de coleta, transporte e pré-tratamento } \\
\text { dos resíduos. Alta carga tributária }\end{array}$ \\
\hline Ecológico & $\begin{array}{l}\text { Substituição do metano gerado por fontes } \\
\text { fósseis por CO2, eliminação de fumação } \\
\text { particulada e baixa geração de cinza a qual } \\
\text { pode ser utilizada como fertilizante. Des- } \\
\text { carte adequado dos resíduos. Baixa gera- } \\
\text { ção de odores na queima }\end{array}$ & $\begin{array}{l}\text { Dependendo da composição (matéria- } \\
\text {-prima) pode gerar cinzas e fumaça }\end{array}$ \\
\hline Legal & $\begin{array}{l}\text { Adequação a legislação. Evita a necessidade } \\
\text { de licenças ambientais (lenha). Implanta- } \\
\text { ção da Política Nacional de Resíduos Sóli- } \\
\text { dos (PNRS) }\end{array}$ & $\begin{array}{l}\text { Não há legislação específica para o } \\
\text { produto ou incentivos à sua utilização } \\
\text { nesse sentido. Ausência de especifica- } \\
\text { ções técnicas. Ausência de incentivos } \\
\text { governamentais }\end{array}$ \\
\hline Imagem de mercado & $\begin{array}{l}\text { Responsabilidade Socioambiental. Meios } \\
\text { externos para divulgação do produto. For- } \\
\text { te apelo ambiental do produto }\end{array}$ & Não há \\
\hline Logística & $\begin{array}{l}\text { Ocupação por peso dos veículos de trans- } \\
\text { porte do produto final. Facilidade de ma- } \\
\text { nuseio e armazenagem. Limpeza de pátios } \\
\text { e inibição de insetos e pragas. Produto } \\
\text { compacto e homogêneo }\end{array}$ & $\begin{array}{l}\text { Alto custo para transporte de matéria- } \\
\text {-prima (maravalha, serragem) por lon- } \\
\text { gas distâncias. Elevado volume e baixo } \\
\text { peso. Sazonalidade da produção de } \\
\text { resíduos. Resíduos florestais apresen- } \\
\text { tam alto teor de umidade (custos de } \\
\text { secagem). Necessidade de armazenar } \\
\text { os briquetes em local coberto }\end{array}$ \\
\hline Social & $\begin{array}{l}\text { Criação de emprego e renda. Melhoria da } \\
\text { qualidade do solo. Redução da disposição } \\
\text { incorreta de resíduos }\end{array}$ & $\begin{array}{l}\text { Descrença do consumidor quanto ao } \\
\text { produto e à qualidade. Desconheci- } \\
\text { mento da aplicação. Foco do consumi- } \\
\text { dor em preço }\end{array}$ \\
\hline
\end{tabular}




\section{CONSIDERAÇÕES FINAIS}

Esta pesquisa concluiu que os principais motivos para investimento na produção de briquetes são: o aumento de ganho econômico; opção para destinação adequada; redução de dependência de outras organizações para a coleta; e redução do risco de paralisação de atividades.

Quanto às barreiras, estas podem ser compreendidas como a alta carga tributária; a sazonalidade do negócio; falta de informação pelo mercado; investimento elevado em equipamentos, e elevados custos com energia elétrica e manutenção. Foi observada também a utilização de espécies de densidade adequada, granulometria específica do resíduo e índice de umidade baixo. É preferível e indicada a utilização de resíduos de empresas que possuam estufas e que apresentem disponibilidade de matéria-prima própria. A aquisição de resíduos de outras empresas pode elevar o custo do produto final e torná-lo menos competitivo em relação a outras fontes energéticas. Não há incentivos governamentais ao segmento.

Portanto, sugere-se a partir desta pesquisa para que o consumo e investimentos em briquetes sejam incentivados é primordial: (i) políticas públicas de apoio, principalmente devido aos impactos negativos de outras fontes energéticas; (ii) concessão de incentivos fiscais de modo a garantir competitividade do produto; (iii) desenvolvimento de convênios com universidades para incentivar pesquisas no que tange ao adensamento de resíduos; (iv) criação de normatização para o produto de forma a garantir qualidade aos consumidores; e (v) desenvolvimento de regulamentações que coíbam o uso da lenha e intensificação da fiscalização por órgãos públicos da utilização imprópria da mesma.

Este estudo abriu mais uma possibilidade de pesquisa futura. Ainda não foi verificado o motivo de poucas empresas atuarem no ramo da briquetagem, conforme afirmam Felfli et al. (2011) e Sousa (2012), apesar de afirmações na literatura apontarem que o investimento em briquetagem é economicamente viável (FILIPPETTO, 2008; SILVEIRA; LOPES, 2011; TAVARES; TAVARES; MOREIRA, 2015; SILVA; CARNEIRO; LOPES, 2017; SCHMIDT, 2017; MAKASHIMA et al., 2018). Futuras pesquisas, principalmente baseadas em survey, poderão propor respostas a esta pergunta. 


\section{REFERÊNCIAS}

ALMEIDA, A.; SILVA, J.; ANGELO, H. Caracterização do ambiente de negócios para produção de madeira serrada no Brasil e no Canadá. Floresta, v. 41, n. 4, p. 751764, out./dez. 2011.

BARBOSA, L.; PEDRAZZI, C.; FERREIRA, E.; SCHNEID, G.; WILLE, V. Avaliação dos resíduos de uma serraria para a produção de celulose kraft. Ciência Florestal, v. 24, n. 2, p. 491-500, abr./jun. 2014.

BHATTACHARYA, S. C.; SETT, S.; SHRESTHA, R. M. State of the art for biomass densification. Energy Sources, v. 11, n. 3, p. 161-182, maio 2007.

BORGHI, M. M. Efeito da granulometria na avaliação dos briquetes. 2012. $47 \mathrm{f}$. Trabalho de Conclusão de Curso (Bacharelado em Engenharia Industrial Madeireira) - Universidade Federal do Espírito Santo, Jerônimo Monteiro, 2012.

BRASIL. Lei n⿳ 12.305, de 2 de agosto de 2010. Institui a Política Nacional de Resíduos Sólidos; altera a Lei no 9.605, de 12 de fevereiro de 1998; e dá outras providências. Disponível em: http://www.planalto.gov.br/ccivil_03/_ato2007-2010/2010/ lei/112305.htm. Acesso em: 27 fev. 2018.

BRITO, L. S.; CUNHA, M. E. T. Reaproveitamento de resíduos da indústria moveleira. Unopar Científica Ciências Exatas e Tecnológicas, v. 8, n. 1, p. 23-26, nov. 2009.

BUZIN, P. J. W. K. Desenvolvimento de briquetes autorredutores a partir de carepas de processamento siderúrgico para utilização em forno elétrico a arco. 138 f. 2009. Dissertação (Mestrado em Engenharia) - Universidade Federal do Rio Grande do Sul, Porto Alegre, 2009.

CAETANO, M.; DEPIZZOL, D.; REIS, A. Analysis of solid waste management and improvement proposal: a case study in carpentry of Cariacica-ES. Gestão \& Produção, v. 24, n. 2, p. 382-394, 2017.

CARVALHO, E. A.; BRINCK, V. Briquetagem. In: LUZ, A. B.; SAMPAIO, J. A.; ALMEIDA, S. L. M. (org.). Tratamento de minérios. Rio de Janeiro: CETEM-MCT, 2004. p. 613-636.

CORTEZ, L. A. B.; LORA, E. E. S.; GÓMEZ, E. O. Biomassa para energia. Campinas: Ed. da Unicamp, 2008. 
DALÓLIO, F.; SILVA, J.; BAÊTA, F.; TINÔCO, I.; CARNEIRO, A. Nota técnica: Cama de frango e resíduo moveleiro: alternativa energética para a zona da mata mineira. Revista Engenharia na Agricultura, v. 25, n. 3, p. 261-271, maio/jun. 2017.

DANTAS, A. P.; SANTOS, R. R.; SOUZA, S. C. O briquete como combustível alternativo para a produção de energia. In: CONGRESSO BRASILEIRO DE GESTÃO AMBIENTAL, 3., 2012, Goiânia. Anais [...]. Goiânia: IBEAS, 2012. v. 1, p. 1-5.

DIAS, J. D. S. et al. Produção de briquetes e péletes a partir de resíduos agrícolas, agroindustriais e florestais. Brasília: Embrapa Agroenergia, 2012. 130 p.

DIAS, J. J. Utilização da biomassa: avaliação dos resíduos e utilização de pellets em caldeiras domésticas. 2002. 112 f. Dissertação (Mestrado) - Universidade Técnica de Lisboa. Lisboa, 2002.

DUTRA, R. I. J. P.; NASCIMENTO, S. M.; NUMAZAWA, S. Resíduos de indústria madeireira: caracterização, consequências sobre o meio ambiente e opções de uso. Revista Científica Eletrônica de Engenharia Florestal, v. 3, n. 5, jan. 2005.

EMERHI, E. A. Physical and combustion properties of briquettes produced from sawdust of three hardwood species and different organic binders. Advances in Applied Science Research, v. 2, n. 6, p. 236-246, 2011.

EMPRESA DE PESQUISA ENERGÉTICA. Balanço Energético Nacional 2017: ano base 2016. Rio de Janeiro: EPE, 2017.

FAGUNDES, H. A. V. Produção de madeira serrada e geração de resíduos do processamento de madeira de florestas plantadas no Rio Grande do Sul. 2003. 173 f. Dissertação (Mestrado) - Universidade Federal do Rio Grande do Sul, Porto Alegre, 2003.

FARAGE, R. M. P. Aproveitamento dos resíduos lignocelulósicos gerados no polo moveleiro de Ubá para fins energéticos. 2009. 105 f. Dissertação (Mestrado) - Universidade Federal de Ouro Preto, Ouro Preto, 2009.

FARAGE, R. M. P. et al. Avaliação do potencial de aproveitamento energético dos resíduos de madeira e derivados gerados em fábricas do polo moveleiro de Ubá MG. Ciência Florestal, v. 23, n. 1, p. 203-212, jan./mar. 2013.

FELFLI, F. F.; ROCHA, J. D.; FILIPPETTO, D.; LUENGO, C. A.; PIPPO, W. A. Biomassbriquetting and its perspectives in Brazil. Biomass and Bioenergy, v. 35, n. 1, p. 
236-242, jan. 2011.

FILIPPETTO, D. Briquetagem de resíduos vegetais: viabilidade técnico-econômica e potencial de mercado. 2008. 74 f. Dissertação (Mestrado em Planejamento de Sistemas Energéticos) - Universidade Estadual de Campinas, Campinas, 2008.

GENTIL, L. V. B. Tecnologia e economia do briquete de madeira. 2008. 195 f. Tese (Doutorado em Engenharia Florestal) - Universidade de Brasília, Brasília, 2008.

GONÇALVES, J. E. Avaliação energética e ambiental de briquetes produzidos com rejeitos de resíduos sólidos urbanos e madeira de Eucalyptus grandis. 104 f. 2010. Tese (Doutorado em Agronomia) - Universidade Estadual Paulista Júlio de Mesquita Filho, Botucatu, 2010.

GOUVÊA, A. et al. Estudo da adição da lignina kraft nas propriedades mecânicas dos briquetes de resíduos da indústria moveleira. Ciência Florestal, v. 27, n. 3, p. 1029-1036, 2017.

HERBST, É. Diagnose da gestão de resíduos sólidos no setor moveleiro da RMC e contribuições para o projeto da central de resíduos. 2011. 130 f. Dissertação (Mestrado) - Universidade Federal do Paraná, Curitiba, 2011.

IBÁ - INDÚSTRIA BRASILEIRA DE ÁRVORES. Relatório IBA 2015. Disponível em: http://iba.org/pt/noticias/gestao-de-residuos-solidos. Acesso em: 22 mar. 2018.

LIMA, E. G.; SILVA, D. A. Resíduos gerados em indústrias de móveis de madeira situadas no pólo moveleiro de Arapongas-PR. Floresta, v. 35, n. 1, p. 105-116, jan./ abr. 2005.

LUZ, S. O. C.; SELLITTO, M. A.; GOMES, L. P. Environmental performance measurement supported by a multicriterial approach: a case study in a manufacturing operation in the automotive industry. Gestão \& Produção, v. 13, p. 557-570, set./ dez. 2006.

MAKASHIMA, G. T. et al. Briquetes produzidos a partir do aproveitamento de resíduos provenientes do aterro de resíduos inertes da cidade de Sorocaba. Revista Brasileira de Energias Renováveis, v. 7, n. 2, p. 231-243, 2018.

MORAIS, D. M. Briquetes de resíduos ligno-celulósicos como potencial energético para a queima de blocos cerâmicos: aplicação em uma indústria de ce- 
râmica vermelha que abastece o Distrito Federal. 2007. 265 f. Tese (Doutorado em Estruturas e Construção Civil) - Universidade de Brasília, Brasília, 2007.

MURAKAMI, F.; SULZBACH, A.; PEREIRA, G. M.; BORCHARDT, M.; SELLITTO, M. How the Brazilian government can use public policies to induce recycling and still save money? Journal of Cleaner Production, v. 96, n. 1, p. 94-101, jul. 2015.

MWAMPAMBA, T. H.; OWEN, M.; PIGAHT, M. Opportunities, challenges and way forward for the charcoal briquette industry in Sub-Saharan Africa. Energy for Sustainable Development, v. 17, p. 158-170, apr. 2013.

OLIVEIRA, T. L. Análise energética e exergética de biomassas como fonte energética sustentável em um forno elétrico a arco. 2013. 126 f. Dissertação (Mestrado) - Universidade Federal de Ouro Preto, Ouro Preto, 2013.

PANWAR, V.; BRASAD, B.; WASEWAR, K. L. Biomass residue briquetting and characterization. Journal of Energy Engineering, v. 137, n. 2, p. 108-114, nov. 2011.

PARASHAR, A.; SINGH, H. K. A case study: energy generation using biomass briquettes in brick kiln of Dholphur, Rajasthan, India. Progress in Science and Engineering Research Journal, v. 2, n. 4-6, p. 81-85, 2014.

PROTÁSIO, T. D. P.; BUFALINO, L.; MENDES, R. F.; RIBEIRO, M. X.; TRUGILHO, P. F.; LEITE, E. R. D. S. Torrefação e carbonização de briquetes de resíduos do processamento dos grãos de café. Revista Brasileira de Engenharia Agrícola e Ambiental, v. 16, n. 11, p. 1252-1258, nov. 2012.

QUIRINO, W. F. Briquetagem de resíduos lignocelulósicos. [s. l.]: LPF/IBAMA, 2005.

QUIRINO, W. F.; BRITO, J. O. Características e índice de combustão de briquetes de carvão vegetal. Brasília: IBAMA, 1991.

RAMÍREZ-GÓMEZ, A. et al. Values for particle-scale properties of biomass briquettes made from agroforestry residues. Particuology, v. 12, p. 100-106, feb. 2014.

ROSÁRIO, L. M. Briquetagem visando utilização de resíduos de uma serraria. 2011. 37 f. Monografia - Departamento de Engenharia Florestal, Universidade Federal do Espírito Santo, Jerônimo Monteiro, 2011. 
ROY, M. M.; CORSCADDEN, K. W. An experimental study of combustion and emissions of biomass briquettes in a domestic wood stove. Applied Energy, v. 99, p. 206-212, nov. 2012.

SCHMIDT, Jéssica Andressa. Estudo da viabilidade de produção de briquetes de biomassa de madeira na região de Lages. 2015. 34 f. Relatório de Estágio (Graduação) - Universidade do Planalto Catarinense, Lages, 2015.

SCHUTZ, F. C. A.; ANAMI, M. H.; TRAVESSINI, R. Desenvolvimento e ensaio de briquetes fabricados a partir de resíduos lignocelulósicos da agroindustria. Inovação e Tecnologia, v. 1, n. 1, p. 3-8, 2010.

SELLITTO, M. A.; KADEL JR., N.; BORCHARDT, M.; PEREIRA, G. M.; DOMINGUES, $\mathrm{J}$. Rice husk and scrap tires co-processing and reverse logistics in cement manufacturing. Ambiente \& Sociedade, v. 16, n. 1, p. 141-162, jan./mar. 2013.

SELLITTO, M. A. Assessment of the effectiveness of green practices in the management of two supply chains. Business Process Management Journal, v. 24, p. 2348, jan./fev. 2018.

SHARIFI, V.; SWITHENBANK, J.; JAMSA-JOUNELA, S. A study on the dynamic combustion behavior of a biomass fuel bed. Fuel, v. 135, p. 468-481, nov. 2014.

SILVA, C. A. Estudo técnico-econômico da compactação de resíduos madeireiros para fins energéticos. 2007. 68 f. Dissertação (Mestrado) - Universidade Estadual de Campinas, Campinas, 2007.

SILVA, J. W. F.; CARNEIRO, R. A. F.; LOPES, J. M. Da biomassa residual ao briquete: viabilidade técnica para produção de briquetes na microrregião de Dourados. Revista Brasileira de Energias Renováveis, v. 6, n. 4, p. 624-646, 2017.

SILVA, J.; CARNEIRO, R.; OLIVEIRA, S.; LOPES, J. Biomassa residual agrícola para produção de briquetes na microrregião de Dourados-MS. Cadernos de Agroecologia, v. 11, n. 2, p. 235-246, 2016.

SILVA, V. S.; GARCIA, C. A.; SILVA, C. M. O destino do bagaço da cana-de-açúcar: um estudo a partir das agroindústrias sucroalcooleiras do Paraná. Revista em Agronegócio e Meio Ambiente, v. 3, n. 1, p. 59-76, jan./abr. 2010.

SRIVASTAVA, N. S. L.; NARNAWARE, S. L.; MAKWANA, J. P.; SINGH, S. N.; VAHORA, $\mathrm{S}$. Investigating the energy use of vegetable market waste by briquetting. Renewab- 
le Energy, v. 68, p. 270-275, aug. 2014.

TAVARES, M. A. M. E.; TAVARES, S. R. L.; MOREIRA, I. T. A produção de briquetes para amenizar a pressão antrópica sobre o bioma caatinga na região do Baixo-Açu Potiguar. Holos, Natal, v. 5, p. 256-270, set./out. 2015.

TAVARES, M. A. M. E. Estudo da viabilidade da produção de briquete e seus possíveis impactos sobre o meio ambiente e o mercado de trabalho da regiáo do Baixo-Açu, RN. 2013. 246 f. Dissertação (Mestrado em Economia) - Universidade Federal da Paraíba, João Pessoa, 2013.

TUOTO, M. Projeto PNUD BRA 00/20. Curitiba: MMA, 2009.

YIN, R. Estudo de caso: planejamento e métodos. Porto Alegre: Bookman, 2010.

YUSOFF, S. Renewable energy from palm oil e innovation on effective utilization of waste. Journal of Cleaner Production, v. 14, p. 87-93, dec. 2006.

ZERBINATTI, O. E.; SILVA, A. B.; PEREIRA, A. J.; MIRANDA, J. M. Briquetagem de resíduos de cafeeiro conduzido no sistema safra zero. Semina: Ciências Agrárias, v. 35, n. 3, p. 1143-1152, maio/jun. 2014.

Recebido em: 12/01/2018 Aceito em: 08/08/2018 